This is an Accepted Manuscript of

S. V. Suraci and D. Fabiani, "Life modeling analysis of Silane crosslinked polyethylene cables for nuclear applications"

in:

2020 IEEE 3rd International Conference on Dielectrics (ICD), 2020, pp. 61-64

The final published version is available online at:

https://doi.org/10.1109/ICD46958.2020.9341894

Rights / License:

The terms and conditions for the reuse of this version of the manuscript are specified in the publishing policy. For all terms of use and more information see the publisher's website.

This item was downloaded from IRIS Università di Bologna (https://cris.unibo.it/)

When citing, please refer to the published version. 


\section{Life modeling analysis of Silane crosslinked polyethylene cables for nuclear applications}

\author{
Simone Vincenzo Suraci \\ LIMES (Laboratory of Innovative Materials \\ for Electrical Systems) - DEI \\ University of Bologna \\ Bologna, Italy \\ simone.suraci@unibo.it
}

\author{
Davide Fabiani \\ LIMES (Laboratory of Innovative Materials \\ for Electrical Systems) - DEI \\ University of Bologna \\ Bologna, Italy \\ davide.fabiani@unibo.it
}

\begin{abstract}
In this article, the evaluation of the electrical and mechanical properties of short XLPE cables subjected to radiochemical aging is reported. From these results, an initial multiphysics life modelling approach is presented. In particular, the proposed modelling of the electrical properties (here complex permittivity) showed to successfully assess of the health of the cables through non-destructive techniques putting the basis of a diagnostic method for cable management on site.
\end{abstract}

Keywords-Cable life modelling, XLPE aging, Gamma radiation, nuclear $\mathrm{LV}$ cables, low-voltage cables, dielectric spectroscopy, mechanical properties, electrical properties.

\section{INTRODUCTION}

It has been estimated that each nuclear power plant (NPP) owns about $1500 \mathrm{~km}$ of instrumentation and control (I\&C) low voltage (LV) cables $[1,2]$. Since the service life of these cables is designed to be about 40 or 60 years and power plants utilities are trying to extend the life of the plants up to other 40 years, a lot of research efforts have been spent focusing on the condition monitoring of LV cables through nondestructive testing techniques, in order to set end-of-life criteria which could take into account also the cable electrical properties.

In this framework, the European H2020 TeaM Cables Project aims at providing NPP operators with a novel methodology for efficient and reliable NPP cable aging management. Among the aims of this Project, the building of a life model for the abovementioned LV cables is a key phase for predicting the health of the cables.

The aim of modelling the cable properties with aging is to estimate either the residual life of in-service cables or the expected life of new cables as closely as possible. Modelling electrical properties, in particular, raises the possibility to evaluate the health of the cables through non-destructive techniques $[2,3]$.

Up to now, the health of NPPs cable systems with aging has been evaluated from their mechanical properties [1, 2, 3], particularly their elongation-at-break $(\mathrm{EaB})$. This because cables must be sufficiently ductile to prevent any deep damage to the cable itself which can bring to breakdown of the insulation and cause its crisis.

End-of-life criterion is usually set at $50 \%$ absolute value of the elongation-at-break of the cable specimen. This criterion had its basis in a report issued by EPRI [1] in 2005. In this report dozens of extruded LV cable specimens have been aged and mechanically tested before and after a Loss of Coolant Accident (LOCA) simulation. This simulation is very important and common inside NPPs since it ensures, in specific areas, the possibility of the cable system (particularly safety cables) to deliver signal or data after a nuclear accident. It has been demonstrated that all the cables which withstood a LOCA simulation owned an EaB equal or higher $50 \%$ absolute.

Despite this, other Research institutes suggest a more conservative approach setting the EoL criterion at $50 \%$ of the relative $\mathrm{EaB}$ [2] which can correspond to, e.g. for a $\mathrm{PE}$ extruded cable, about $150 \%$ of the absolute EaB. However, this criterion, which is the one used in this study, presuppose the $\mathrm{EaB}$ value for the new cable to be known, which is not always available. Similar approach is given by the IEC 602162 , which suggests the $50 \%$ relative elongation as end-of-life criterion for the evaluation of thermal endurance characteristics of cables.

In the recent years, electrical properties, in particular dissipation factor (Tan $\delta$ ), gained more and more interest by some researchers since they appeared to be suitable for the evaluation of health condition of the cable with aging in a nondestructive way $[3,6]$.

Aim of this article is on the one hand to present the correlation between these two techniques, on the other hand to introduce a life model which could possibly scale up the actual diagnostics methods for cables, laying the basis for a novel analysis methodology of cable insulation health.

\section{MATERIALS AND MethodS}

\section{A. Specimens}

The samples here analyzed are low-voltage I\&C coaxial cables with XLPE insulation used in NPPs, especially designed for the project. Morphology of the investigated cables is reported in Fig. 1. Specimens are made of five concentric parts (reported in the caption).

The primary insulation, in particular, is a silane crosslinked polyethylene stabilized with 1 phr of primary antioxidant (phenol-based) and 1 phr of secondary antioxidant (thioetherbased).

Each cable specimen is about $50 \mathrm{~cm}$ long.

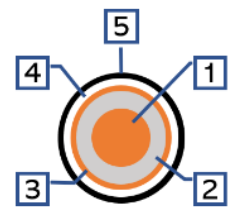

Fig. 1. Multilayer structure of coaxial cables under investigation. (1) Conductor - Copper, (2) Primary insulation - XLPE, (3) Polymeric film PET, (4) Shielding - Copper wire braid, (5) Sheath - Low smoke zero halogen 


\section{B. Accelerated aging}

Three different dose rates, namely: high, medium and low dose rate corresponding to 400,60 and $7 \mathrm{~Gy} / \mathrm{h}$ respectively, have been chosen to perform cable aging. In particular, radiothermal ageing was performed in the Panoza (Medium and Low dose rate) and Roza (High Dose Rate) facility at UJV Rez, Czech Republic, through a ${ }^{60} \mathrm{Co} \gamma$-ray source.

Aging conditions are summarized in Tab. 1.

TABLE I. AGING CONDITIONS

\begin{tabular}{|l|c|c|c|}
\hline \multirow{2}{*}{$\begin{array}{c}\text { Aging } \\
\text { type }\end{array}$} & \multicolumn{3}{|c|}{ Aging properties } \\
\cline { 2 - 4 } & Dose rate (Gy/h) & Sampling time (h) & $\begin{array}{c}\text { Total absorbed } \\
\text { dose }(\boldsymbol{k G y})\end{array}$ \\
\hline Low & 7 & 3456 & 81 \\
\hline Medium & 60 & 864 & 286 \\
\hline High & 400 & 167 & 334 \\
\hline
\end{tabular}

At this stage, few samplings for the low dose rate (LDR) aging are present, so that the LDR modelling can result as not completely reliable.

\section{B. Dielectric spectroscopy measurements}

Complex permittivity and tan $\delta$ have been investigated through a Novocontrol Alpha dielectric analyzer with applied voltage of $3 \mathrm{~V}_{\mathrm{rms}}$ in the frequency region $10^{6}-10^{-2} \mathrm{~Hz}$. Tests have been performed in oven at $50{ }^{\circ} \mathrm{C}$.

Tan $\delta$, known also as dissipation factor (DF), is defined by [3-6]:

$$
\tan \delta=\frac{\omega \varepsilon^{\prime \prime}+\sigma}{\omega \varepsilon^{\prime}}
$$

where $\varepsilon^{\prime}$ is the real part of permittivity defined as the dielectric constant of the material, $\varepsilon$ ' is the imaginary part of permittivity related to the dielectric losses of the material, $\omega$ is the angular frequency of the wave and $\sigma$ is the conductivity of the material [3-6].

Considering high values of frequencies is possible to neglect the contribution given by the conductivity and claim that the dissipation factor is directly proportional to the dielectric losses of the material.

\section{Mechanical tensile stress measurements}

Tested samples were aged as whole $0.5 \mathrm{~m}$ long cable with cable ends protected against oxidation. Insulation tubes of the length of $100 \mathrm{~mm}$ were prepared from these cables after the end of aging. For tensile test, the calibrated Instron 3366 machine equipped with pneumatic grips with smooth steel surfaces was used. The gripping and also the gauge length of tubular insulation specimens was $30 \mathrm{~mm}$ and the test speed of $50 \mathrm{~mm} / \mathrm{min}$ was applied.

\section{MODELLING APPROACH}

The modelling approach presented in this study is based on the "Assessment and management of ageing of major nuclear power plant components important to safety: Incontainment instrumentation and control cables" report by the IAEA [2].

This approach allows the building of a curve representing the maximum dose a cable can bare before reaching the crisis of the cable itself, for a given dose rate. In other words, it is made up of points indicating the total doses required to reach a specific end point criterion.

As a result, the curve is built in a log/log plot featuring the Dose Rate (DR) values and the so called Doses Equivalent to Damage (DED). This latter is defined as the dose, one per each dose rate investigated, at which the EoL point is reached. This plot is found to be linear in a log-log plot for the XLPE insulated cables, enabling extrapolation of DED to lower dose rates [2]. The end point doses are given by

$$
D E D=K \cdot D R^{n} \quad(\mathrm{kGy})
$$

where DR is the dose rate; $\mathrm{K}$ and $\mathrm{n}$ are empirical constants specific to the material tested. The constant $\mathrm{n}$ is usually in the range 0 to 0.3 .

In order to build such plot, an EoL criterion must be assessed. As presented in the introduction, up to date there is no standard or set rule which claims an unique EoL criterion based on $\mathrm{EaB}$. In this work $50 \%$ of the relative $\mathrm{EaB}$ has been chosen as EoL point for two main reasons: the proximity of $50 \%$ relative with the experimental data and the conservativeness of this approach (which can be suggested for e.g. safety cables).

The model proposed is based on some assumptions:

- Test data must be obtained in isothermal conditions;

- $\quad$ The highest value of analyzed DR is such that homogenous oxidation conditions are achieved;

- $\quad$ The dose rates analyzed must be at least three, in order to build a reliable curve.

Among the various limitations this approach can face such as the extrapolation to dose rates within the thermally dominated aging region, there is the fundamental obstacle that this modelling approach is based on mechanical destructive tests which are usually not representative of the entire cable system and in general difficult to perform on real cables.

The novelty of this work lays on the possibility to scale up the actual standard (i.e. EaB tests) using nondestructive electrical techniques.

To do so, the trend of the electrical properties, one per each aging condition considered, plotted versus the total absorbed dose, allows the extrapolation of the EoL criterion based on the electrical property. Indeed, the value of $\tan \delta$ corresponding to the DED obtained through the EaB measurements analyses, will be the end point of the cable dissipation factor.

These $\tan \delta$ values at a fixed frequency namely: Dissipation Factor at DED (DF $\left.\mathrm{DED}_{\mathrm{D}}\right)$, one per each dose rate considered, allows a new $\tan \delta$ vs DR curve to be built following the power law:

$$
D F_{D E D}=c \cdot D R^{s}
$$

Also in this case, DR is the dose rate; $c$ and $s$ are empirical constants specific to the material tested for a given temperature.

Among the frequency values, $100 \mathrm{kHz}$ has been chosen since it is showed in literature $[3,4]$ to be representative of the cable aging conditions and, consequently, possibly usable as an aging marker for cables based on electrical nondestructive measurements.

The curve built this way for a given cable-type could allow the evaluation of the cable system health, in terms of electrical fulfillment of its work, entirely through electrical testing technique which are, as presented, nondestructive. 


\section{EXPERIMENTAL RESULTS AND DISCUSSION}

\section{A. Tensile stress}

Figure 2 displays the behavior of the relative $\mathrm{EaB}$ as a function of the total dose for the three aging conditions considered. Relative $\mathrm{EaB}$ have been calculated by the ratio between the actual $\mathrm{EaB}$ and the $\mathrm{EaB}$ of the unaged cable.

Once plotted, a linear regression line was built in order to obtain the end-of-life point corresponding at $\mathrm{EaB} / \mathrm{EaB}_{0}=0.5$. Moreover, in order to keep the uncertanty and the confidence intervals coming from these measurements, other two lines were built corresponding to the upper (lower) limit of the highest (lowest) $\mathrm{EaB} / \mathrm{EaB}_{0}$ value obtained, respectively. So that every measurement obtained is inside the built confidence interval. However due to page contraints only one graph reporting the values of the median for the three aging conditions are reported in Figure 2.

Unfortunately, due to the severe conditions, it was not possible to remove the primary insulation from the conductor in the third and fourth aging period of the low dose rate. For this reason, only the first two points for the tensile measurements are here reported.

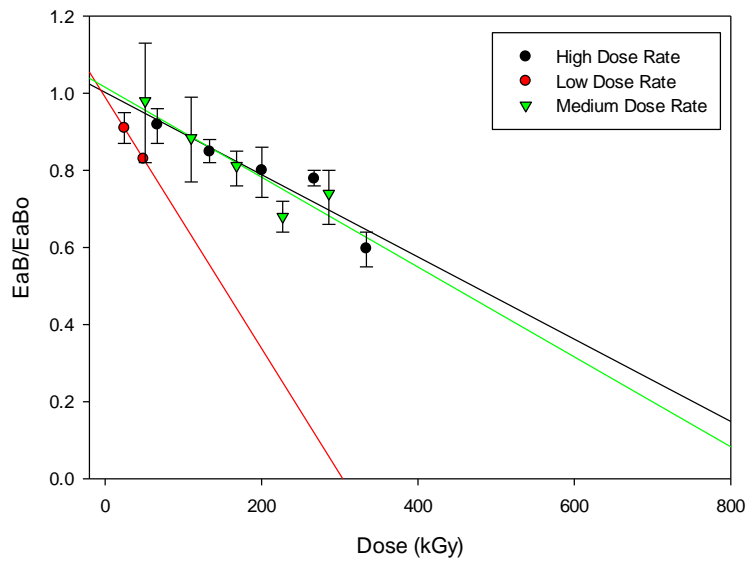

Fig. 2. Relative EaB as a function of the total absorbed dose for Low dose rate (red), Medium Dose Rate (green) and High Dose Rate (black) aging.

\section{B. Dielectric spectroscopy}

Figure 3 shows the trend of the dielectric losses $(\tan \delta)$ at $100 \mathrm{kHz}$ as a function of the total dose for the analyzed aging conditions.

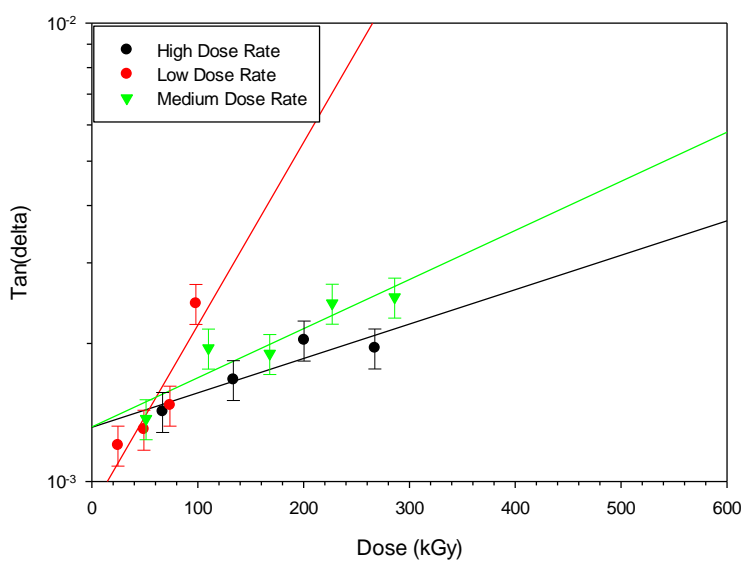

Fig. 3. Tan $\delta$ at $100 \mathrm{kHz}$ as a function of the total dose for the analyzed aging conditions (Linear regression lines).
As expected, the values of the dielectric losses raise with the increase of the aging period. From Figure 3 it is evident that the dose rates do have an impact on the response of the material during aging, indeed, different dose rates result in different values of $\tan \delta$, for a fixed absorbed dose. This discrepancy is more and more evident as we increase the total dose (or the aging time). In particular, the lower the dose rate, the higher the dielectric losses. This behavior could be imputed, on the one hand, to the effect of aging time which is way longer on lower dose rates with respect to the higher dose rate here considered; on the other hand to the fact that a lower dose rate brings to a more homogenous degradation of the insulation matter $[4,7]$. Indeed, with higher dose rates (e.g. high dose rate - black curve) the degradation results to be not homogenous but stronger in the external layer, leading to smaller variations in the bulk of the polymer matter and thus to very little variation of the tan $\delta$ which is a bulk property.

\section{MODELLING RESUltS AND DisCUSSION}

\section{A. Acquisition of the DED value}

The Dose values, and contextually the upper and lower limits, corresponding to $\mathrm{EaB} / \mathrm{EaB}_{0}=0.5$ are selected and used for the application of the above described power law method. Hence, the Dose (kGy) values equivalent to the EoL criterion, namely the Dose Equivalent to Damage (DED) are reported in Tab.2.

TABLE II. END-OF-LIFE DOSE VALUES

\begin{tabular}{|l|c|c|c|}
\hline $\begin{array}{c}\text { Aging } \\
\text { type }\end{array}$ & $\begin{array}{c}\text { Regression } \\
\text { DED (kGy) }\end{array}$ & $\begin{array}{c}\text { Upper limit } \\
(\mathbf{k G y})\end{array}$ & $\begin{array}{c}\text { Lower limit } \\
(\mathbf{k G y})\end{array}$ \\
\hline Low & 150 & 165 & 141 \\
\hline Medium & 444 & 583 & 310 \\
\hline High & 466 & 535 & 370 \\
\hline
\end{tabular}

These results are finally plotted in a log DED vs $\log$ DR plot, reported in Fig. 4.

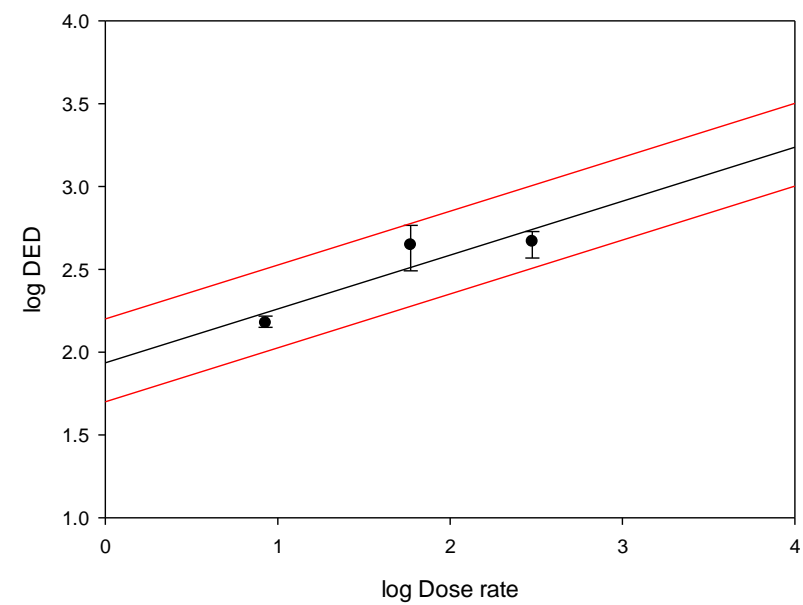

Fig. 4. Extrapolation of Dose equivalent to Damage for the considered cable. $(\log / \log$ scale)

Hence, the regression line, representative of the doses corresponding to the crisis of the cable for a given dose rate, is obtained through the following equation:

$$
D E D=K \cdot D R^{n}=147 \cdot D R^{0.2}(k G y)
$$


where DR is the considered Dose Rate.

\section{B. Application to the electrical measurements}

Once obtained DED values from (3) it is possible to graphically obtain the end-of-life point in terms of Tan $\delta$, which will finally give the value of dielectric losses corresponding to the failure of the material from Figure 3.

Even in this case, the values of $\tan \delta$ corresponding to the upper and the lower limit of the DED are obtained and registered in order to keep into account the measurements uncertainties.

Similarly, the procedure can be replicated for the other two dose rates. Therefore, the Tan $\delta$ values corresponding to the DED are reported in Tab. 3 .

TABLE III. END-OF-LIFE TAN $\quad$ VALUES

\begin{tabular}{|c|c|c|c|}
\hline $\begin{array}{l}\text { Aging } \\
\text { type }\end{array}$ & Tanס at DED & Tand upper limit & $\begin{array}{c}\text { Tand lower } \\
\text { limit }\end{array}$ \\
\hline Low & 0.00351 & 0.00415 & 0.00323 \\
\hline Medium & 0.00397 & 0.0053 & 0.0028 \\
\hline High & 0.002891 & 0.00331 & 0.0025 \\
\hline
\end{tabular}

\section{Modelling of the electrical properties}

The $\tan \delta$ values corresponding to the DED are finally plotted in a tan $\delta$ vs DR plot, reported in Fig. 6. Note that the values are plotted in a $\log / \log$ (absolute value) scale since applying the logarithm to the tan $\delta$ values result in a negative amount.

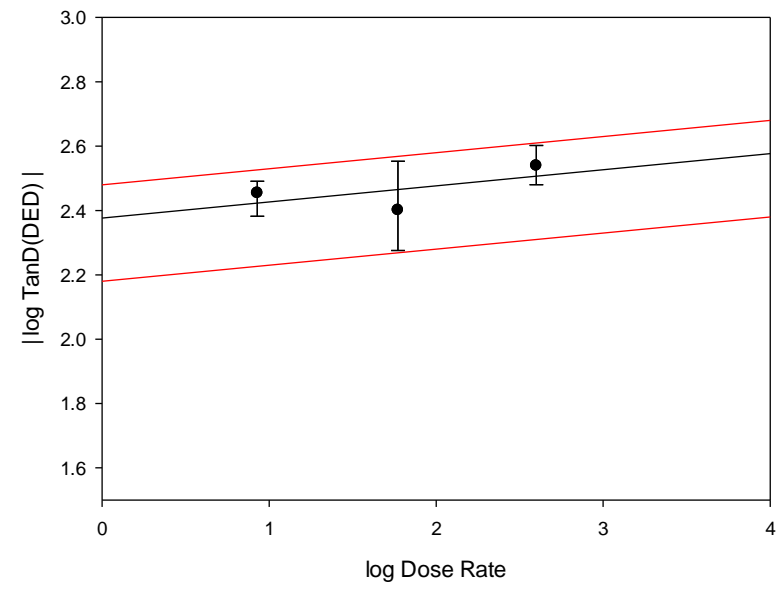

Fig. 5. Dielectric losses lifecurve as a function of Dose Rates for the coaxial cable considered. (log/ inverse log scale)

The regression line built represents the values of $\tan \delta$ the cable owns at DED corresponding to the crisis of the cable.

Finally, it is possible to obtain for the coaxial cable here considered, the equation representative the $\tan \delta$ End-of-Life curve is:

$$
D F_{D E D}=0.004 \cdot D R^{-0.04}(-)
$$

where DR is the considered Dose Rate.

Here equation (4) describes the end-of-life points in terms of dielectric properties of the analyzed system. Due to this, it is reasonable to claim that if the actual value of DF is lower than the DF value corresponding to DED, precisely if $D F \leq D F_{D E D}$ is verified, the cable owns an EaB higher than $50 \%$ rel. Consequently, it does not need replacement according to the actual health assessment criteria.

\section{CONCLUSIONS}

The modelling approach presented in this article could be considered as a first attempt at setting an EoL criterion based on non-destructive techniques, which can be a substantial upgrading of the actual cable managements inside NPPs. It allows the evaluation of the dissipation factor corresponding at the crisis of low voltage cables for NPPs applications, for a given the dose rate. Furthermore, it permits the estimation of the life of the cable through only non-destructive testing techniques, scaling-up the actual standard for cable monitoring, which uses destructive testing techniques e.g. tensile stresses that are often not easy to perform on site and are not always representative of the entire cable system.

On the other side, $\tan \delta$ measurements take into account the entire cable system giving information about the health and the electrical applicability of the polymer insulation analyzed.

To conclude, ideally, the possibility to build a life curve for each cable system could allow the diagnostics of cable systems in an easy and nondestructive manner.

Future works on this topic include the definition of a new quantity, which is related to the cable strength (CS) in terms of cumulative dose. This quantity will aim at the extrapolation of the residual life of the considered cable for a given set of stressors (e.g. radiation or thermal) and application time, knowing only the values of the presented cable constants.

\section{ACKNOWLEDGMENT}

Authors are grateful to Vit Placek and Hakim Janah for their support and useful discussion on the topic.

Authors are also grateful to the Radiation Chemistry and Environmental Qualification Department, UJV Rez (ÚJV Řež), Czech Republic for performing the tensile stress and for support and discussion on the topic

The project leading to this application has received funding from the

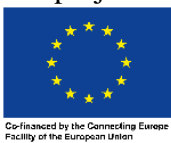
Euratom research and training programme 20142018 under grant agreement No 755183. The authors are grateful to Nexans and UJV for sample preparation and aging.

\section{REFERENCES}

[1] G. Thoman, "Initial Acceptance Criteria Concepts and Data for Assessing Longevity of Low-Voltage Cable Insulations and Jackets," EPRI, available https://www.epri.com/\#/pages/product/1008211

[2] IAEA “Assessment and management of ageing of major nuclear power plant components important to safety", 2000.

[3] S. V. Suraci, D. Fabiani, A. Xu, S. Roland, and X. Colin, "Ageing Assessment of XLPE LV Cables for Nuclear Applications Through Physico-Chemical and Electrical Measurements," IEEE Access, vol. 8, pp. 27086-27096, 2020, doi: 10.1109/ACCESS.2020.2970833.

[4] S. V. Suraci, D. Fabiani, K. Sipilä, and H. Joki, "Filler impact analysis on aging of crosslinked polyethylene for nuclear applications through dielectric spectroscopy," p. 4.

[5] A. K. Jonscher, "Dielectric relaxation in solids," J. Phys. D: Appl. Phys., vol. 32, no. 14, pp. R57-R70, Jan. 1999, doi: 10.1088/00223727/32/14/201.

[6] S. V. Suraci, D. Fabiani, L. Mazzocchetti, and L. Giorgini, "Degradation Assessment of Polyethylene-Based Material Through Electrical and Chemical-Physical Analyses," Energies, vol. 13, no. 3, p. 650, Jan. 2020, doi: 10.3390/en13030650.

[7] B. Bartoníček, V. Plaček, and V. Hnát, "Comparison of degradation effects induced by gamma radiation and electron beam radiation in two cable jacketing materials," Radiation Physics and Chemistry, vol. 76, no. 5, pp. 857-863, May 2007, doi: 10.1016/j.radphyschem.2006.05.011 
